\title{
Hybrid optical-fibre/geopolymer sensors for structural health monitoring of concrete structures
}

\author{
M. Perry ${ }^{1}$, M. Saafi ${ }^{2}$, G. Fusiek ${ }^{3}$, P. Niewczas ${ }^{3}$ \\ ${ }^{1}$ Department of Civil and Environmental Engineering, James Weir Building, \\ University of Strathclyde, Glasgow, G1 1XJ \\ 2 Department of Engineering, Lancaster University, Lancaster, LA1 4YR \\ 3 Department of Electronic and Electrical Engineering, Royal College Building, \\ University of Strathclyde, Glasgow, G1 1XW \\ E-mail: m.perry@strath.ac.uk
}

\begin{abstract}
.
In this work, we demonstrate hybrid optical-fibre/geopolymer sensors for monitoring temperature, uniaxial strain and biaxial strain in concrete structures. The hybrid sensors detect these measurands via changes in geopolymer electrical impedance, and via optical wavelength measurements of embedded fibre Bragg gratings. Electrical and optical measurements were both facilitated by metalcoated optical fibres, which provided the hybrid sensors with a single, shared physical path for both voltage and wavelength signals. The embedded fibre sensors revealed that geopolymer specimens undergo $2.7 \mathrm{~m} \varepsilon$ of shrinkage after one week of curing at $42{ }^{\circ} \mathrm{C}$. After curing, an axial $2 \mathrm{~m} \varepsilon$ compression of the uniaxial hybrid sensor led to impedance and wavelength shifts of $7 \times 10^{-2}$ and $-2 \times 10^{-4}$ respectively. The typical strain resolution in the uniaxial sensor was $100 \mu \varepsilon$. The biaxial sensor was applied to the side of a concrete cylinder, which was then placed under $0.6 \mathrm{~m} \varepsilon$ of axial, compressive strain. Fractional shifts in impedance and wavelength, used to monitor axial and circumferential strain, were $3 \times 10^{-2}$ and $4 \times 10^{-5}$ respectively. The biaxial sensor's strain resolution was approximately 10 $\mu \varepsilon$ in both directions. Due to several design flaws, the uniaxial hybrid sensor was unable to accurately measure ambient temperature changes. The biaxial sensor, however, successfully monitored local temperature changes with $0.5^{\circ} \mathrm{C}$ resolution.
\end{abstract}




\section{Introduction}

Smart civil structures typically employ a network of optical or electronic sensors to monitor the root causes of deterioration. Automated measurements of strain, temperature or chemical contamination are used to verify or replace visual inspections, providing more affordable and accurate assessments of structural health [1]. Optical fibre sensors such as fibre Bragg gratings (FBGs) have already been used to monitor the health of bridges, buildings, tunnels and foundations [2]. Lightweight, one-dimensional and robust, these sensors detect changes in strain via the modulated properties of guided light. FBG sensors can be serially multiplexed and then surface mounted or embedded into civil structures to provide quasi-distributed, highresolution measurements of strain and temperature $[3,4,5]$.

The technical challenge of providing affordable, long-term packaging and attachment methods for fibre sensors often presents a barrier to their widespread use [6]. Electronic sensors, on the other hand, provide a more established, cost-effective route for health evaluation, albeit with a lower resolution and accuracy [7]. Mechanical strain can be monitored, for instance, via variations in the electrical behaviour of surfacemounted piezos [8]. Geopolymer binders, created by combining fly ash with alkaline activators, are just one example of a class of chemically stable, lowshrinkage piezoresistive materials, highly suited to civil applications. Geopolymer binders provide excellent adhesion to concrete structures [9], and once cured, can be used to detect strain and temperature through changes in electrical impedance [10].

While both sensing schemes have their limitations, hybrid electronic/optical sensors can use the advantages of both sensor architectures to enhance measurement capability [11, 12]. However, as hybrid schemes often require separate electrical and optical connections, the sensor network's complexity and installation time may be increased.

In this paper, we present and characterise two designs for a hybrid geopolymer/FBG sensor. The first sensor is a simple cylindrical cell for monitoring local temperature and uniaxial strain. The second sensor is an adhesive patch, designed to monitor the temperature and biaxial strain of a neighbouring concrete structure. In both cases, metal-coated optical fibres and polymer-coated FBGs are embedded into a geopolymer matrix. While fibre optic sensors have been encapsulated in geopolymers before [13], in this work, the geopolymer substrate is functionalised so that it can also act as a secondary electronic strain/temperature sensor. As both components of the sensor can measure strain and temperature, the sensors can verify their own readings to improve measurement confidence and reduce the likelihood of common mode failures.. Furthermore, as metal-coated fibres can carry both electrical current and light, they provide a single, shared pathway for electrical and optical interrogation, eliminating the need for secondary connections. The geopolymer's adhesive properties, meanwhile, provide a convenient, ruggedised packaging and attachment method for the FBGs, ensuring good strain transfer from the neighbouring concrete body. Both sensor designs can be multiplexed, allowing the schemes to be scaled up to monitor large civil structures. To our knowledge, this is the first time a truly hybrid fibreoptic/geopolymer sensor has been proposed.

\section{Sensing principles}

\subsection{Fibre Bragg gratings}

A fibre Bragg grating (FBG) is a 10-20 mm long periodic modulation in the refractive index of an optical fibre, shown schematically in Figure 1. This modulation is written into photosensitive optical fibres by side-illuminating them with an ultraviolet laser. When broadband light guided within the fibre meets the grating, a narrow distribution of wavelengths are back-reflected towards the light source [14]. The centre of this wavelength distribution, termed the Bragg peak, $\lambda_{B}$, undergoes linear fractional shifts as strain, $\varepsilon$, and temperature changes, $\Delta T$, are applied to the FBG:

$\frac{\Delta \lambda}{\lambda}=K_{\varepsilon} \varepsilon+K_{T} \Delta T$

Here $K_{\varepsilon}$ and $K_{T}$ are the strain and temperature sensitivity of the FBG, respectively. The effects of strain and temperature can be decoupled by using two adjacent FBGs with different sensitivities - a sensor at $\lambda_{1}$ and a local reference at $\lambda_{2}$. If the reference is packaged so that it is, for example, immune to external strain, $\left(K_{\varepsilon 2}=0\right)$, then the weighted, relative wavelength shift, $\Delta \lambda_{r e l}$, is used to find the strain in the sensor:

$\varepsilon=\frac{1}{K_{\varepsilon 1}}\left(\frac{\Delta \lambda_{1}}{\lambda_{1}}-\frac{K_{T 1}}{K_{T 2}} \frac{\Delta \lambda_{2}}{\lambda_{2}}\right)=a \Delta \lambda_{r e l}$ 


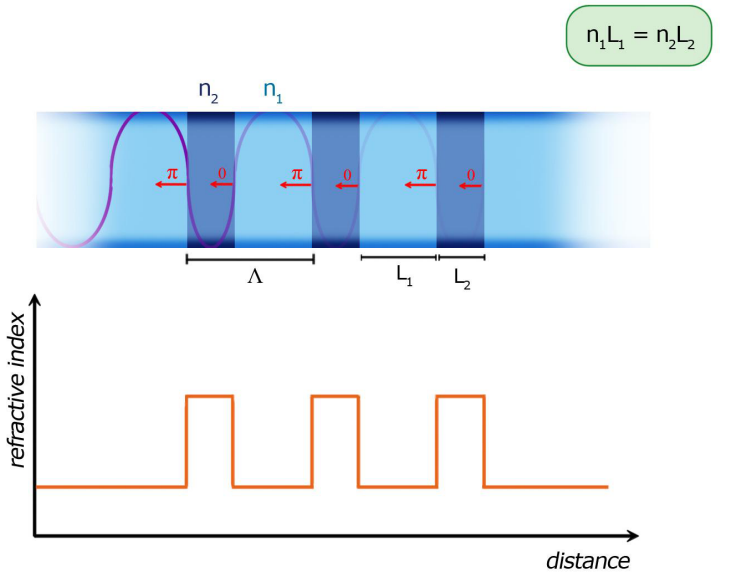

Figure 1. The periodicity, $\Lambda$, and refractive indices, $n_{1}$ and $n_{2}$, of a fibre Bragg grating's modulation both change with strain and temperature. This causes shifts in the back-reflected Bragg wavelength, $\lambda_{B}$.

\subsection{Geopolymer impedance}

Geopolymer binders can be synthesised by combining fly ash with alkaline activators. The resulting geopolymer gel is an adhesive, which can be cured over several hours at elevated temperatures to form an amorphous solid. After curing, residual electrolytic alkaline solutions within the pores of geopolymers allow them to act as fast ionic conductors [15]. Geopolymers therefore display enhanced electrical conductivities, similar to those of semiconducting materials.

When electrodes with a contact area, $A$, are used to apply an alternating current, $I$, across a geopolymer specimen, the measured voltage, $V$, is affected by changes in the specimen's impedance, $Z$ :

$\frac{V}{I}=Z=\frac{\rho L}{A}$

where $\rho$ and $L$ are the resistivity and length of the geopolymer material between the electrodes. In analogy with the wavelength shifts of an FBG sensor, measurands which affect impedance, such as strain and temperature, induce fractional changes in the measured voltage:

$\frac{\Delta V}{V}=\frac{\Delta Z}{Z}=p(\varepsilon)+q(\Delta T)$

Here, the functions $p$ and $q$ may be non-linear and describe the geopolymer sensor's strain and temperature sensitivity.

Impedance is complex, so its magnitude, $|Z|$, and phase angle, $\phi$, can be used to calculate the resistance, $R$, and reactance, $X$, of a geopolymer specimen at a given circuit frequency, $f$ :

$Z=|Z| e^{j \phi}=R+j X=R+\frac{j}{2 \pi f C}$ where $C$ is the sensor's capacitance. In practice, high-frequency alternating currents are applied to geopolymer sensors during impedance measurements. This reduces the effects of capacitance and false polarisation potentials at the sensor electrodes [16].

\section{Hybrid sensor design and characterisation}

\subsection{Fabrication technique}

In this work, the geopolymer binder was made by combining $72 \mathrm{wt} \%$ low-calcium, class-F fly ash, with 20 wt\% sodium silicate solution $\left(\mathrm{Na}_{2} \mathrm{SiO}_{3}\right.$ with 29.4 $\mathrm{wt} \% \mathrm{SiO}_{2}, 14.7 \mathrm{wt} \% \mathrm{NaO}_{2}$ in water) and $8 \mathrm{wt} \%$ of 10 $\mathrm{M}$ sodium hydroxide solution.

Optical fibre components were commercially sourced. Single-mode optical fibres coated in copperalloy (Cu1300) and acrylate (SMF28) were used to address the sensors. FBGs were written in bare singlemode optical fibre and then re-coated in acrylate.

3.1.1. Uniaxial sensor Figure 2a shows the arrangement of the first hybrid sensor, designed to measure uni-axial strain and temperature. An acrylated coated FBG was cleaved and then spliced, at either end, to lengths of metal-coated fibre. The splices and the FBG section were enveloped in a plastic splice-protector, with a stainless steel rod. This shielded the FBG and bare spliced sections from mechanical shear and bending.

The encapsulated FBG was embedded into a $27 \mathrm{~mm}$ diameter, $50 \mathrm{~mm}$ long tube of geopolymer gel and allowed to cure and solidify at $42{ }^{\circ} \mathrm{C}$ for 3-7 days before stress testing. After curing, the exposed metalised-fibre connections were passed through electrical insulation and a steel part to allow radial egress from the cylindrical sensor, as shown in Figure 3a. This allowed axial forces to be applied to the sensor cell without breaking the fibre connections.

3.1.2. Biaxial sensor Figure $2 \mathrm{~b}$ shows the arrangement used for the second hybrid sensor, designed to measure bi-axial strain and temperature in concrete. As with the uniaxial sensor, FBG sections were spliced to metalised adressing fibres to form the optical and electrical connections. In this case, a temperaturereference FBG at $\lambda_{2}$ was fabricated by encapsulating the FBG in a two layers of copper capillaries, filled with thermally conducting grease. This configuration aimed to eliminate the transfer of external mechanical strain to the FBG.

The temperature reference and a second, acrylatecoated strain-sensing FBG (at wavelength $\lambda_{1}$ ) were circumferentially aligned to the side of a concrete cylinder, shown in Figure 3b. The FBGs and metalised fibre connections were then bonded using geopolymer 
a)

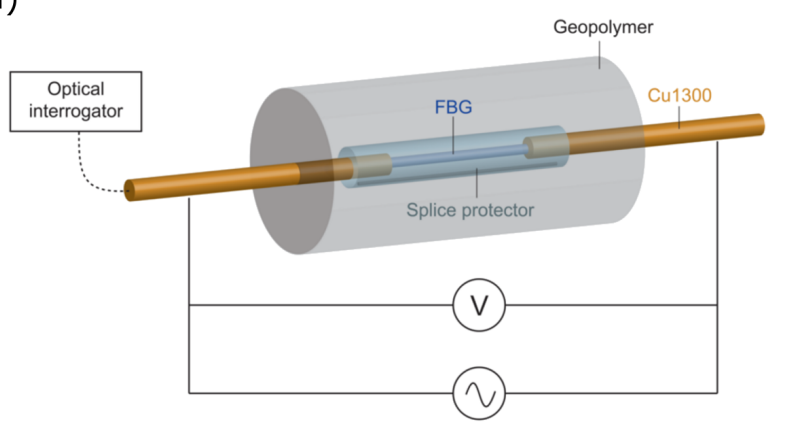

b)

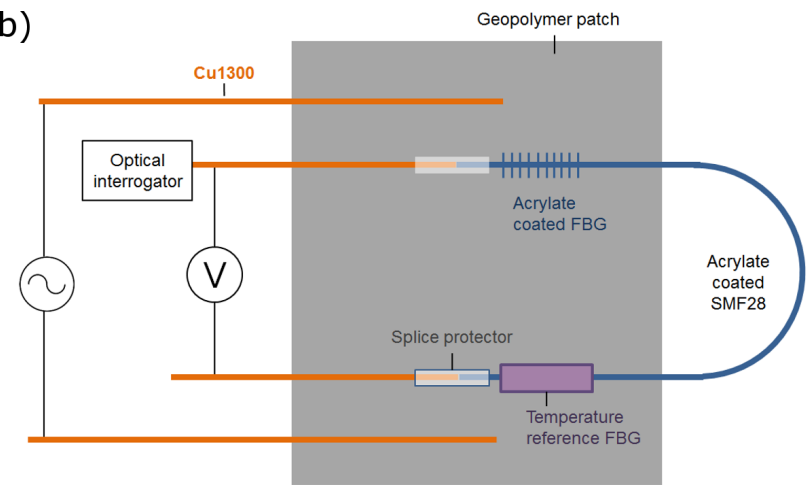

Figure 2. Schematics of the a) uniaxial and b) biaxial hybrid FBG/geopolymer sensors, showing locations of interrogators, FBGs and copper-alloy coated fibres (Cu1300).

adhesive and allowed to cure at $42{ }^{\circ} \mathrm{C}$ for 3-7 days before stress testing. The geopolymer patch spanned a surface of $60 \mathrm{~mm} \times 40 \mathrm{~mm}$ and had a thickness of 2 $\mathrm{mm}$.
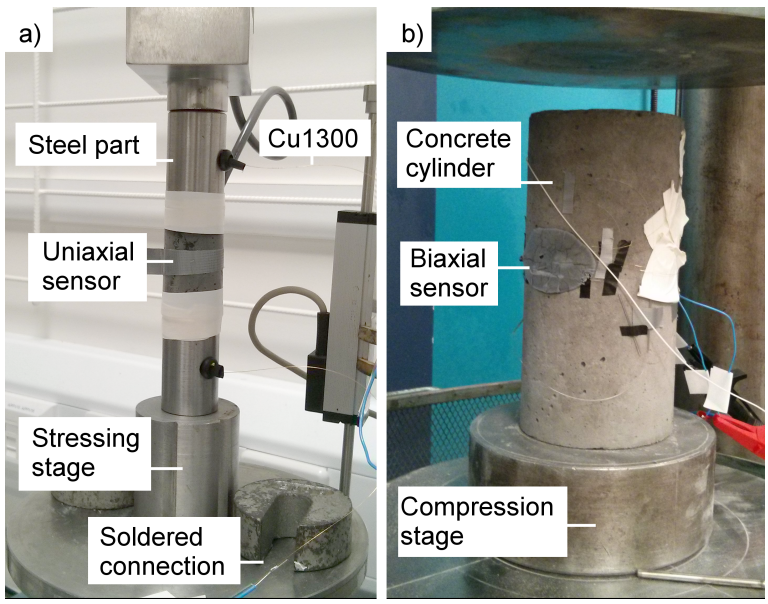

Figure 3. Photographs of a) uniaxial and b) biaxial hybrid sensors, located on their respective compression stages.

After curing, the concrete cylinder (diameter $10 \mathrm{~cm}$, height $20 \mathrm{~cm}$ ) was subjected to axial compressive loading. The compressive force resulted in strain changes which were monitored via the impedance of the geopolymer patch. Meanwhile, radial strains (a consequence of the Poisson effect) were monitored by the circumferential strain-sensing FBG. The temperature-reference FBG provided temperature compensation for the strain measurements.

\subsection{Interrogation}

Current sources applied alternating currents between $5 \mu \mathrm{A}$ and $200 \mu \mathrm{A}$ to the geopolymer sensors at frequencies between $100 \mathrm{~Hz}$ and $5 \mathrm{kHz}$. As shown in Figure 2a, the alternating current was applied serially over the uniaxial sensor, via wires soldered to the metalised fibre. The voltage was monitored over the same path using a data acquisition card (typical voltage noise was $2 \mathrm{mV}$ ). The biaxial sensor used the same electrical hardware, but configured as shown in Figure 2b to allow four-terminal impedance measurements. The alternating current was applied across the outer-probes and the voltage was measured over the inner probes. While this set up is more complicated, separation of the electrodes allows for more accurate impedance monitoring as it reduces contact and lead resistances.

FBG wavelengths between $1520 \mathrm{~nm}$ and 1580 $\mathrm{nm}$ were monitored using a commercial interrogator (wavelength resolution of $10 \mathrm{pm}$ ) and peak detection algorithm. Wavelength and voltage changes in the hybrid sensors were monitored simultaneously at a measurement frequency of $1 \mathrm{~Hz}$.

\subsection{Characterisation methods}

In this work, the embedded FBG was monitored during curing of the uniaxial sensor. While this was not strictly required for sensor characterisation, it allowed the geopolymer binder's solidification and shrinkage during curing to be investigated.

After curing, the complex impedance of the uniaxial hybrid sensor was also investigated. Alternating voltages (amplitude $1.5 \mathrm{~V}$, frequency $f=100 \mathrm{~Hz}$ to $5 \mathrm{kHz}$ ) were applied to the uniaxial sensor and the impedance and phase angle were measured using a Resistance-Capacitance-Inductance (RCL) meter.

Both uniaxial and biaxial hybrid sensors were heated in an oven to characterise their temperature sensitivity. The responses of the FBG wavelengths and the geopolymer impedance were interrogated, with ambient temperatures monitored using a separate thermocouple.

To characterise strain sensitivity for the uniaxial sensor, axial forces up to $1 \mathrm{kN}$ were applied directly 
to the cylindrical geopolymer specimen using a small compression tester, shown in Figure 3a. For the biaxial sensor, a large compression tester (Figure 3b) applied axial loads as high as $100 \mathrm{kN}$ (equivalent to $50 \%$ compressive strength) to the concrete cylinder.

\section{Results}

\subsection{Uniaxial sensor}

4.1.1. Shrinkage Figure 4 shows the strain measured by the FBG, embedded in the uniaxial sensor, during one week of curing at $42{ }^{\circ} \mathrm{C}$. The rate of shrinkage initially increased, reaching a maximum after approximately 45 hours, before decreasing after 100 hours. The final shrinkage measured after 7 days was approximately $2.7 \mathrm{~m} \varepsilon$. This may not be an accurate representation of actual shrinkage in the specimen, as the measurement is affected by the changing strain transfer between the FBG, the splice protector and the solidifying geopolymer. Nevertheless, the shrinkage behaviour is consistent with the theory that water within the geopolymer is used in a set of accelerating geopolymerisation reactions [17]. Surface dehydration in the dry oven environment is also thought to have had a large impact. Although low calcium fly-ash was used in the work presented here, shrinkage rates and magnitudes show broad agreement with previous work using high-calcium fly ash [18].

4.1.2. Impedance Figure 5 shows Nyquist and Bode plots obtained for the uniaxial sensor during impedance characterisation using the RCL metre. As expected, the impedance is larger and mainly dominated by resistance at low frequencies. Reactance dominates beyond driving frequencies of $f=1 \mathrm{kHz}$. The peak

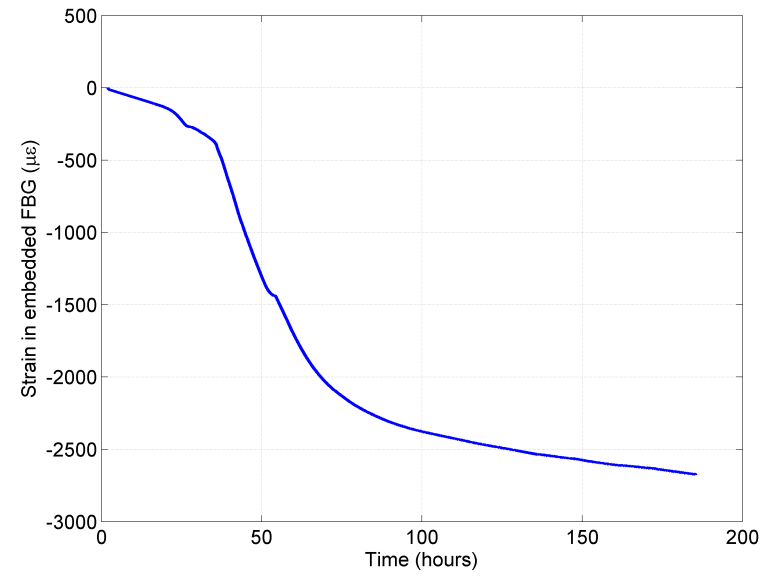

Figure 4. Shrinkage strain measured by an FBG embedded in the uniaxial geopolymer sensor during 185 hours of curing at 42 ${ }^{\circ} \mathrm{C}$. in impedance at around $3.2 \mathrm{kHz}$ may be due to a resonance in the circuit, either in the geopolymer specimen itself or in the contacts and leads. The low resolution of the RCL meter used in this work did not allow resonances to be investigated further.

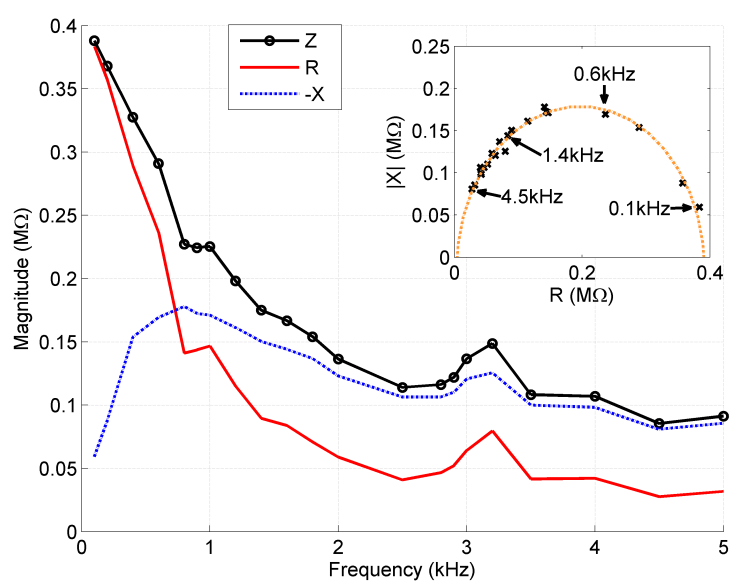

Figure 5. Nyquist (and inset, Bode) plot of the impedance of the uniaxial hybrid sensor at $1.5 \mathrm{~V}$.

When designing a geopolymer sensor, it is important to select an intermediate driving frequency for measurement because:

- operating at high frequencies reduces capacitive effects, preventing polarisation potentials from confounding voltage measurements [16];

- reducing the frequency increases the total impedance so that safe levels of electrical current are able to provide a high-quality, measurable voltage signal.

Equation (5) and Figure 5 suggest that, between $500 \mathrm{~Hz}$ and $1500 \mathrm{~Hz}$, capacitance is reduced to approximately $1 \mathrm{nF}$ and a high overall impedance of $0.2 \mathrm{M} \Omega$ is maintained. At these frequencies, the biaxial sensor displayed a similar impedance of approximately $0.1 \mathrm{M} \Omega$. As such, alternating current driving frequencies were set to $1 \mathrm{kHz}$ during the temperature and strain characterisation in this work.

4.1.3. Temperature sensitivity The temperature response of the uniaxial sensor's impedance and wavelength are shown in Figure 6. Note that the wavelength response has been multiplied by $10^{3}$ for clarity. Both variables increase during heating, but the impedance shifts are more pronounced.

This result could be interpreted as being due to thermal expansion of the sensor, as length increases cause positive shifts in $\lambda_{B}$ and $Z$. This may be the case for the wavelength shifts, but the low thermalexpansion of the geopolymer cannot account for such 


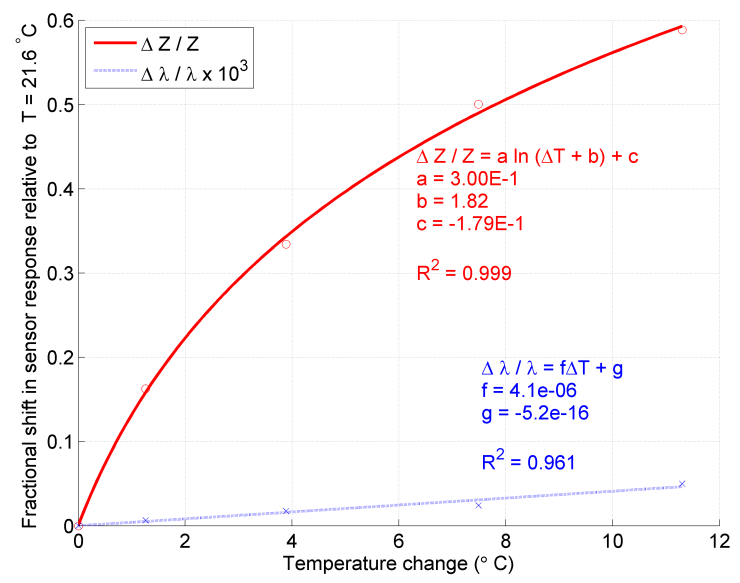

Figure 6. Temperature sensitivity of the FBG and geopolymer components of the hybrid uniaxial sensor. The FBG's wavelength response has been multiplied by $10^{3}$ for clarity.

large, non-linear shifts in impedance [19]. Indeed, as geopolymers are fast ionic conductors, heating should lead to a reduced impedance, as charge carriers become more mobile at elevated temperatures $[15,20]$. It is therefore suggested that the impedance shifts shown in Figure 6 are dominated by enhancements in resistivity, caused by the heating of electrical contacts and leads. The results for the biaxial sensor in Section 4.2.1 support this hypothesis - these four-terminal measurements were less dependent on contact/lead resistances and revealed a negative correlation between impedance and temperature.

4.1.4. Strain sensitivity Figure 7 shows the uniaxial sensor's fractional shifts in wavelength and impedance during a compression test. Wavelength shifts have been multiplied by $10^{3}$. The relationship between the applied compressive strain and sensor's response is shown Figure 8. Here, the applied strain was calculated using measurements from a linear variable differential transformer (LVDT). As shown, a $2 \mathrm{~m} \varepsilon$ compressive strain leads to fractional shifts in impedance and wavelength of $7 \times 10^{-2}$ and $-2.2 \times 10^{-4}$ respectively.

While the wavelength response is linear with strain, Figure 7 shows that there is some wavelength drift, especially at high loads. This is due to creep and relaxation in the system, particularly at the interface between the splice-protector and the geopolymer. The FBG's strain sensitivity, independent of any coating and packaging, is $K_{\varepsilon}=0.78$ [7]. As such, the gradient of the strain response, shown in Figure 8, suggests that the strain transfer from the geopolymer to the FBG is $\alpha=0.10 / 0.78 \approx 13 \%$. The low strain transfer is likely due to the impact of the stainless steel rod and thick plastic sheathing of the splice protector. Usually, the

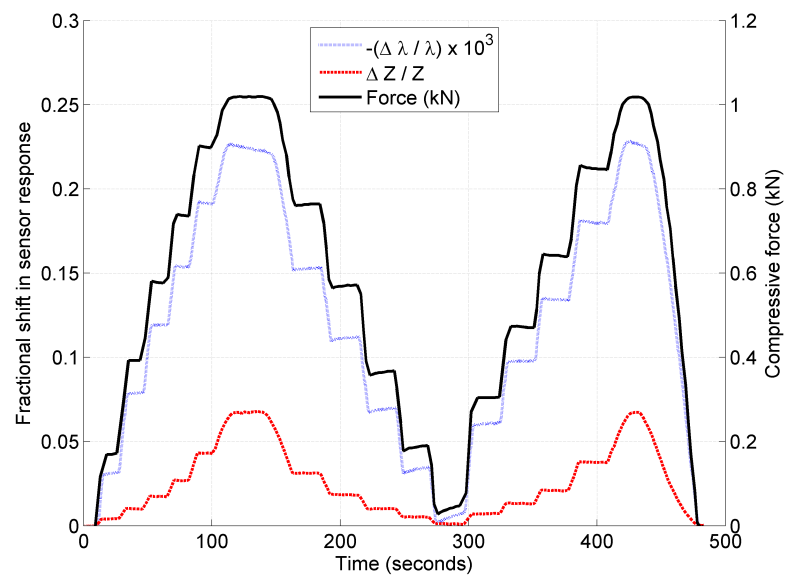

Figure 7. Shifts in the impedance and wavelength of the uniaxial hybrid sensor as forces are step-changed up to $1 \mathrm{kN}$. The FBG's wavelength response has been multiplied by $10^{3}$ for clarity.

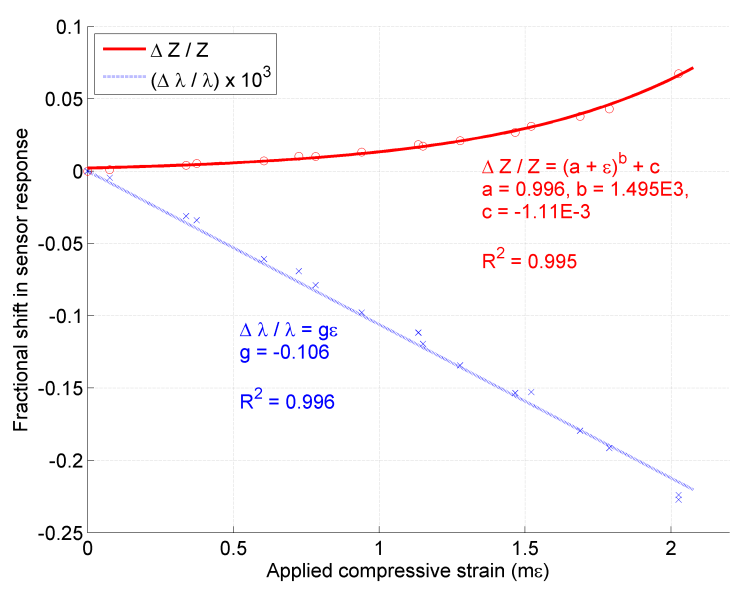

Figure 8. Strain sensitivity of the FBG and geopolymer components of the hybrid uniaxial sensor. The FBG's wavelength response has been multiplied by $10^{3}$ for clarity.

$10 \mathrm{pm}$ resolution of the interrogation system provides strain measurements with $8 \mu \varepsilon$ resolution, but this low strain transfer value reduces the FBG strain resolution to approximately $60 \mu \varepsilon$.

The impedance response of the sensor is nonlinear, but repeatable and less prone to creep. The nonlinearity may have occured because the geopolymer's impedance is sensitive to the formation of micro-cracks and the compressive load was large enough to cause some level of failure. While noise in the measured voltage signal is a reasonably constant $2 \mathrm{mV}$, the nonlinear response of the impedance leads to a variable strain resolution. Below $0.5 \mathrm{~m} \varepsilon$, the strain resolution was $100 \mu \varepsilon$, but above $1 \mathrm{~m} \varepsilon$, resolution was improved to $40 \mu \varepsilon$. 


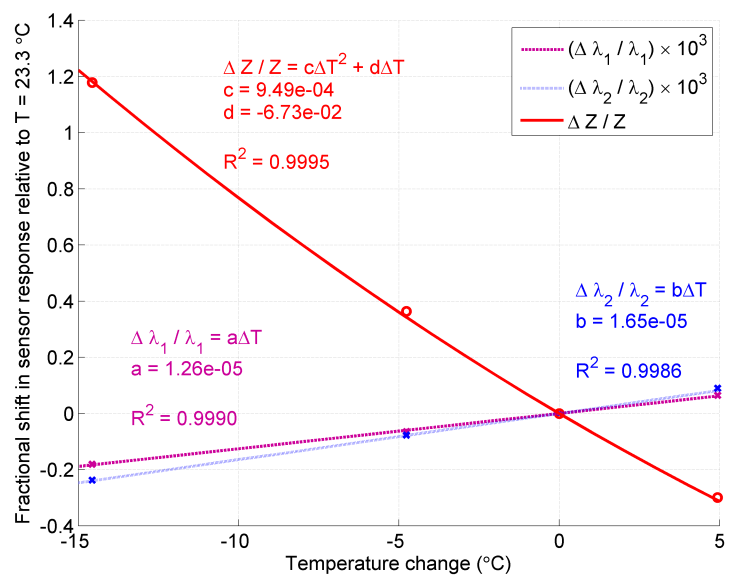

Figure 9. Temperature sensitivity of the FBG and geopolymer components of the hybrid uniaxial sensor. FBG wavelength responses have been multiplied by $10^{3}$ for clarity.

Note that temperature compensation could not be applied to the uniaxial sensor during this experiment, due to the issues with temperature characterisation discussed in Section 4.1.3. The effects were minimal as ambient temperatures were reasonably constant over the short-term compression test.

\subsection{Biaxial sensor}

4.2.1. Temperature sensitivity The temperature response of the biaxial sensor is shown in Figure 9. The sensor $\left(\lambda_{1}\right)$ and temperature-reference $\left(\lambda_{2}\right)$ FBGs both undergo positive wavelength shifts as ambient temperature is elevated. Their different temperature sensitivities arise due to differences in packaging. The temperature-reference FBG was able to monitor ambient temperatures with a resolution of $0.5{ }^{\circ} \mathrm{C}$. The impedance of the geopolymer shows a negative correlation with temperature. This agrees with the current understanding that geopolymers behave as fast ionicconductors, as discussed in Section 4.1.3. Voltage noise suggests that impedance measurements have a temperature resolution equivalent to $0.1{ }^{\circ} \mathrm{C}$.

4.2.2. Strain sensitivity The biaxial sensor's response to a $100 \mathrm{kN}$ axial compression cycle of the concrete cylinder is shown in Figure 10. Fractional shifts in the impedance and FBG-strain-sensor wavelength were $3 \times 10^{-2}$ and $4 \times 10^{-5}$ respectively. The temperaturereference FBG showed minimal response to the load. The drifts and offsets in the response of the impedance and wavelength are partly due to creep, but also partly due to the low accuracy of the compression tester.

The sensor's overall sensitivity to force in the concrete cylinder is shown in Figure 11. Note that equation (2) is used to find the corrected FBG strain

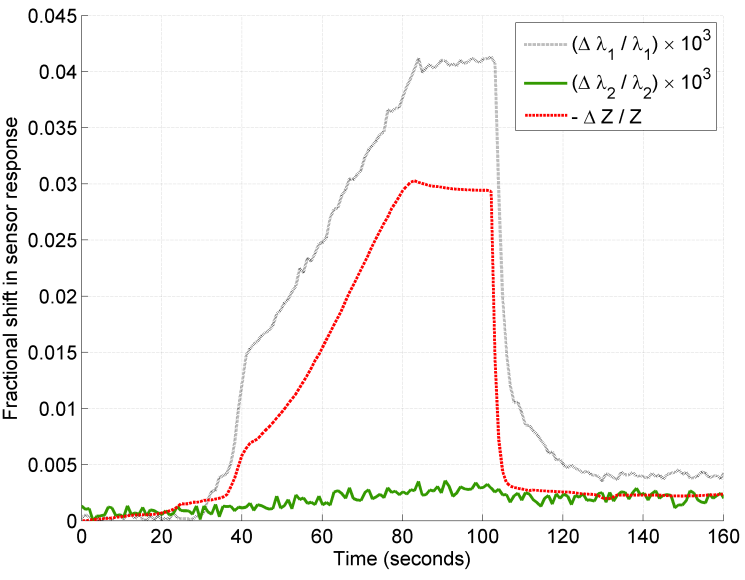

Figure 10. Response of the wavelengths and impedance of the biaxial sensor to a $100 \mathrm{kN}$ compressive load cycle. FBG wavelength responses have been multiplied by $10^{3}$ for clarity.

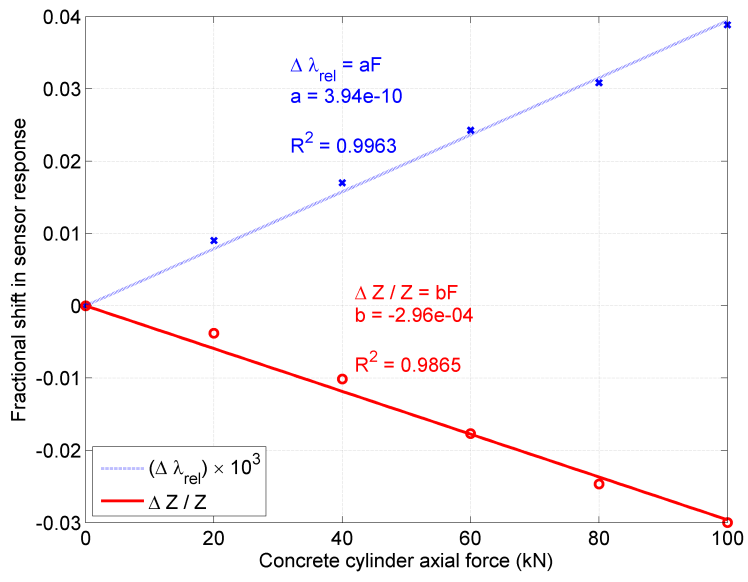

Figure 11. FBG and geopolymer components of the hybrid biaxial sensor shift as the concrete cylinder is compressed. The relative FBG wavelength response has been multiplied by $10^{3}$ for clarity.

response $\Delta \lambda_{\text {rel }}$ shown here. As compression reduces the distance between the electrodes in the geopolymer patch, the impedance decreases as expected. Unlike the impedance response of the uniaxial sensor, very little non-linearity is observed. This may be due to less cracking, as the $100 \mathrm{kN}$ maximum force applied to the cylinder induces smaller compressive strains in the geopolymer patch (approximately $0.6 \mathrm{~m} \varepsilon$, assuming the concrete's Young's modulus is $E_{c}=20$ GPa). Impedance measurements were found to have a resolution equivalent to $10 \mu \varepsilon$.

The Poisson effect causes expansion of the concrete cylinder's circumference, causing the strainsensing FBG to undergo a positive, linear wavelength shift as expected. The gradient of the FBG system's 
strain response, $a$, shown in Figure 11 can be used to estimate the strain transfer from the concrete cylinder, $\varepsilon_{c i r c}$, to the sensor FBG, $\varepsilon_{F B G}$, via:

$\Delta \lambda_{\text {rel }}=K_{\varepsilon} \varepsilon_{F B G}=a \frac{E_{c} D}{4 \nu} \varepsilon_{c i r c}$

where $\nu=0.2$ and $D=10 \mathrm{~cm}$ are the Poisson ratio and diameter of the concrete cylinder respectively. Equation (6) therefore suggests that the strain transfer is $\alpha=\frac{\varepsilon_{\text {circ }}}{\varepsilon_{F B G}} \approx 80 \%$. This high strain transfer value arises because there is no splice protector surrounding the FBG and becase the concrete cylinder is large compared to the geopolymer layer. The value is in good agreement with previous studies which have found wellbonded, surface-mounted fibre sensors should have a strain transfer of 85-95\% [21]. The high strain transfer allows the FBGs to measure circumferential strain with a resolution of $10 \mu \varepsilon$.

\section{Discussion}

In this work, two proof-of-concept designs have been demonstrated for hybrid geopolymer/FBG sensors. The confinement of light in the optical fibre and the low alternating currents of $5-200 \mu \mathrm{A}$ make the sensors inherently safe - an important factor for civil applications.

Overall, the biaxial sensor's measurement capabilities were better than those of the uniaxial sensor. The biaxial sensor demonstrated superior temperature compensation and a linear strain response, due to the use of four-terminal impedance monitoring and more suitable packaging for the FBGs. The reduced thickness of the geopolymer patch in the biaxial sensor also allowed the FBGs to monitor ambient temperature changes more accurately. Nevertheless, both of the prototype sensor designs could be improved by addressing the following issues.

\subsection{Shrinkage}

In this work, geopolymer shrinkage in the uniaxial sensor was measured over one week, but it should be noted that shrinkage will continue after this period. After seven days of curing, the reduced shrinkage rate allows the sensor to be used for shortterm or dynamic strain measurements without a large impact on measurement accuracy. However, if long-term measurements are required, curing times should be extended to alleviate shrinkage before sensor deployment. Curing at higher temperatures or adding fibrous materials to the geopolymer are two other methods of reducing shrinkage that may furthermore increase the geopolymer's compressive strength [18].

\subsection{Measurement quality}

In both sensors, the geopolymer substrate and the electrical connections were directly exposed to temperature increases. For the uniaxial sensor, the thermal dependence of the lead and contact resistances dominated the measurement, while the biaxial sensor was able to measure geopolymer impedance independently of these effects. In both cases, the impedance response to temperature changes was almost immediate.

For the wavelength measurements, it was found that the low thermal conductivity of the geopolymer shielded the FBG from heating [22]. The high thermal inertia of both sensor designs raises major issues for temperature compensation, as it means the relative temperature response of the impedance and wavelength are dependent on the rate of the temperature change. The temperature characterisations presented and used in this work were performed over a one hour temperature cycle, as this provided a comparable time frame to strain tests. Long-term elevated temperatures led to a different response, which was furthermore not repeatable, as long-term heating accelerated geopolymer shrinkage and changed the substrate's chemistry.

Improvements to temperature sensitivity could be made by enhancing the geopolymer's thermal conductivity through the use of additives. The thicknesses of the geopolymer components could be reduced to increase the exposure of FBGs to ambient temperatures, but this may also reduce the conductivity and mechanical robustness of the sensors. Any increase in impedance from downsizing could be somewhat reversed by driving the current at a higher frequency, doping the sensors with carbon nanotubes [23], or by using the sensors exclusively in high temperature applications where charge carriers are more mobile. Hermetic packaging of the geopolymer in an electrically insulating package may also prevent water loss, shrinkage, and any variations in impedance caused by changes in local humidity or chemical contamination.

\subsection{Multiplexing}

Both of the hybrid sensor designs naturally lend themselves to multiplexing. The initial Bragg wavelength of an FBG can be customised during writing, so that wavelength-division-multiplexed arrays of FBGs can be used to monitor quasi-distributed strains in large structures with a single interrogation device. Meanwhile, parallel electronic multiplexing using switches may be used to obtain impedance measurements of the geopolymer substrates. Network complexity may be further reduced by using miniturised optical interrogators and wireless impedance-monitoring technolo- 
gies $[24,25]$.

\section{Conclusions}

Prototype hybrid optical-fibre/geopolymer sensors for measuring strain and temperature in civil structures have been designed and characterised for the first time. A simple cell for monitoring local temperature and uniaxial strain was fabricated, along with a second, adhesive sensor for monitoring temperature and biaxial strain in a neighbouring concrete cylinder. In both cases, metal-coated optical fibres provided an electrical connection for measuring the impedance of geopolymer substrates, and an optical pathway for interrogating embedded fibre Bragg grating sensors. The confinement of light in the optical fibre and the use of alternating currents $<200 \mu \mathrm{A}$ amplitude make the sensors inherently safe.

The embedded fibre Bragg gratings revealed that shrinkage in uniaxial geopolymer sensors was $2.7 \mathrm{~m} \varepsilon$ after one week of curing at $42{ }^{\circ} \mathrm{C}$. Complex impedance measurements of the geopolymer demonstrated that sensors could be interrogated at $1 \mathrm{kHz}$ to reduce capacitive effects on impedance measurements.

Temperature characterisation of the uniaxial sensor was unsuccessful due to the high thermal intertia of the geopolymer cell and due to interference of changing contact/lead resistances. As the biaxial sensor was smaller and utilised four-probe impedance monitoring, it could successfully resolve ambient temperatures with $0.5{ }^{\circ} \mathrm{C}$ resolution.

Application of $2 \mathrm{~m} \varepsilon$ compression to the uniaxial hybrid sensor cell led to repeatable impedance and wavelength shifts of $7 \times 10^{-2}$ and $-2.2 \times 10^{-4}$ in geopolymer and fibre components, respectively. Strain transfer from the geopolymer to the fibre was 10 $\%$ and the strain resolution of both components of the sensor was approximately $100 \mu \varepsilon$. The biaxial sensor monitored strain in a concrete cylinder as it was compressed by up to $0.6 \mathrm{~m} \varepsilon$. The fractional shifts in impedance and wavelength, used to monitor axial and circumferential strain, were $3 \times 10^{-2}$ and $4 \times 10^{-5}$ respectively. Strain transfer from the concrete to the fibre sensors was $80 \%$ and the biaxial sensor's strain resolution was $10 \mu \varepsilon$ in both directions.

\section{References}

[1] Chang P C, Flatau A and Liu S C 2003 Structural Health Monitoring 2 257-267

[2] Leung C K Y, Wan K T, Inaudi D, Bao X, Habel W, Zhou Z Ou J, Ghandehari M, Wu H C and Imai M 2013 Materials and Structures $1-36$

[3] Rodrigues C, Félix C, Lage A and Figueiras J 2010 Engineering Structures 32 1993-2002

[4] Billon A, Henault J M, Quiertant M, Taillade F, Khadour A, Martin R P and Benzarti K 2014 EWSHM - 7th
European Workshop on Structural Health Monitoring (Nantes, France)

[5] Perry M, Yan Z, Sun Z, Zhang L, Niewczas P and Johnston M 2014 Nuclear Engineering and Design 268 35-40

[6] Méndez A 2007 Proc. SPIE vol 6619 pp 661905-661905-6

[7] Lee B 2003 Optical Fiber Technology 9 57-79

[8] Park G, Cudney H and Inman D 2000 Journal of Infrastructure Systems 6 153-160

[9] Pacheco-Torgal F, Castro-Gomes J P and Jalali S 2008 Construction and Building Materials 22 154-161

[10] Saafi M, Tang L, Fung J, Rahman M, Sillars F, Liggat J and Zhou X 2014 Smart Materials and Structures 23065006

[11] da Costa Marques Pimentel R, Beirao Barbosa M, Costa N, Ribeiro D, de Almeida Ferreira L, Araujo F and Calcada R 2008 IEEE Sensors Journal 8 1243-1249

[12] Schlavin J, Zagrai A, Clemens R, Black R J, Costa J, Moslehi B, Patel R, Sotoudeh V and Faridian F 2014 Proc. SPIE vol 9064 pp 90640X-90640X-13

[13] He J, Zhang G, Hou S and Cai C 2011 Journal of Materials in Civil Engineering 23 100-109

[14] Hill K O and Meltz G 1997 Journal of lightwave technology $151263-1276$

[15] Cui X M, Zheng G J, Han Y C, Su F and Zhou J 2008 Journal of Power Sources 184 652-656

[16] Saiprasad Vaidya E N A 2011 Smart Structures and Systems 7 27-40

[17] Zuhua Z, Xiao Y, Huajun Z and Yue C 2009 Applied Clay Science 43 218-223

[18] Ridtirud C, Chindaprasirt P and Pimraksa K 2011 International Journal of Minerals, Metallurgy, and Materials 18 100-104

[19] Zuda L and Černý R 2009 Cement and Concrete Composites 31 263-267

[20] Hanjitsuwan S, Chindaprasirt P and Pimraksa K 2011 International Journal of Minerals, Metallurgy, and Materials 18 94-99

[21] Li D, Ren L and Li H 2012 Fiber Optic Sensors (InTech) ISBN 978-953-307-922-6

[22] Duxson P, Lukey G C and van Deventer J S J 2006 Industrial 83 Engineering Chemistry Research 45 77817788

[23] Saafi M, Andrew K, Tang P L, McGhon D, Taylor S, Rahman M, Yang S and Zhou X 2013 Construction and Building Materials 49 46-55

[24] Saafi M, Kaabi L, McCoy M and Romine P 2010 International Journal of Materials and Structural Integrity 41

[25] Trita A, Vickers G, Mayordomo I, van Thourhout D and Vermeiren J 2014 Proc. SPIE vol 9133 pp 91330D91330D-9 